\title{
Attractive effect of different colored sticky traps on Cotarina sp. in mulberry field
}

\author{
Ren Jiequn ${ }^{1, a}$, Chen Li ${ }^{2, b}$, Li Zhinan ${ }^{1, a^{*}}$, Zhang Minghai ${ }^{1, a}$, Yang $\mathrm{Yi}^{1, \mathrm{a}}$ and \\ Zheng Zhangyun ${ }^{1, a}$
}

${ }^{1}$ The Chongqing Three Gorges Academy of Agricultural Science, Wanzhou Chongqing 404155, China

2 Plant Protection Station of Wanzhou, Wanzhou Chongqing 404199, China a601249780@qq.com, b chenli420625@163.com

Keywords: Colored sticky traps; Mulberry; Cotarina sp.; Suspension heights; Attractive effect Abstract. The trapping effect of eight different kinds sticky traps on adult Cotarina sp. were studied in different suspension heights to provide the basis for monitoring and integrated controlling. The results showed that the suspension height and the color of the sticky traps had significant impacts $(P<0.05)$. The adult of Cotarina sp. had certain tendency to the green, blue, purple, turquoise sticky traps. The green sticky traps showed most attractive effect than any other color, followed by the blue sticky traps, while the turquoise and the purple sticky traps received the worst trapping effect. The adults had no taxis to yellow, red, black and white sticky traps. There was a significant difference $(P<0.05)$ in the effect of different suspension height sticky traps on adult Cotarina sp. The number of adults trapped by sticky traps with $1.0 \mathrm{~m}$ away from the ground was the mostest, which the attractive effect was $37.62 \%$. The tendency of adult Cotarina sp. population quantity attractived by different colors of sticky traps with time was consistent. Two population quantity of adults peaks were found by drawing an emergence curve in spring, one reached on 29 March, and the other reached on 3 April.

\section{Introduction}

Cotarina sp. (Cecidomyiidae, Nematocera, Diptera) was commonly known as " the mulberry fruit gall-midge " and the principal pests on mulberry. This insect which parasitized the mulberry small fruit caused the mulberry malformationand the seeds abnormal development ${ }^{[1-3]}$. In recent years, with the continuous development of the fruit mulberry industry, the problem of mulberry diseases and insect pests have become more and more serious, especially the gall morula has become the main pest in fruit mulberry production. At present, the main method for investigating the population of the insect is using sticky traps in the field. In this paper, by comparing the trapping effect between different colored sticky traps and different suspension heights to select the best color sticky traps or the optimum suspension height. It will provide the theoretical basis and technical support for monitoring and integrated controlling of the mulberry fruit gall-midge.

\section{Materials and Methods}

Experimental Material. The experimental site is located in the mulberry demonstration park of The Chongqing Three Gorges Academy of Agricultural Science in Wanzhou District, Chongqing, China. Test treatment period was from 1 March to 28 April 2017. Eight kinds of sticky traps such as yellow, blue, green, red, black, purple, turquoise and white were produced by Sichuan ruijinte technology co. LTD. The sticky traps, with the specification of $25 \mathrm{~cm} \times 30 \mathrm{~cm}$, were spread glue consistently on both sides. The thickness of glue on each side was uniformly. The executive standard was GB/T 24689.4-2009.

\footnotetext{
Fund projects: Chongqing social welfare science and technology innovation project (cstc2016shmszx 80014); Chongqing sericulture industry development subsidy fund project (CQ2017CSE014)

First Author: Ren Jiequn (1989-), woman, research assistant, E-mail: 601249780@ qq.com

*Corresponding author: Li Zhinan, man, research assistant, E-mail: yourslzn@sina.com
} 
Experimental Method. Trapping effect of different colors of sticky traps on adults, yellow, blue, green, red, black, purple, turquoise and white sticky traps were fixed on wooden stalk and settled in Chinese mulberry field; Trapping effects of different heights of sticky traps on adults, sticky traps were fixed on wooden stalk with $0.5 \mathrm{~m}, 1.0 \mathrm{~m}, 1.5 \mathrm{~m}$ away from the ground. The sticky traps were settled with an interval of $2 \mathrm{~m}$, each treatment was repeated three times. The sticky trap was checked every day for a total of six times and was replaced with a new one at each survey. The number of adult Cotarina sp. on different colors and heights of sticky traps was recorded.

Data Analysis. All data were statistically analyzed using Microsoft office 2010 and IBM SPSS 16.0 software. The number of Cotarina sp. was analyzed by using one-way analysis of variance (ANOVA). An LSD test (significance level was $P=0.05$ ) was performed to analyze differences between experimental groups.

Attractant percent $(\%)=$ The number of adults of each treatment $/$ The overall number of adults $\times 100^{[4]}$

\section{Experimental Results and Analysis}

Trapping effect of different colors of sticky traps on adults. There was a significant difference in the effect of yellow, blue, green, red, black, purple, turquoise and white sticky traps on adult of Cotarina sp. ( table 1). The adults had certain tendency to the green, blue, purple, turquoise sticky traps. The attractive percent of green, blue, turquoise and purple sticky traps on Cotarina sp. was $54.46 \%, 27.23 \%, 12.38 \%$ and $5.94 \%$, respectively. Green sticky traps was the most attractive to adult Cotarina sp., followed by was blue sticky traps, while turquoise and purple sticky traps received the worst trapping effect. The adults had no taxis to yellow, red, black, and white sticky traps.

Table 1 Trapping effect of different colors of sticky traps on adult Cotarina sp.

\begin{tabular}{cccc}
\hline $\begin{array}{c}\text { Kind of sticky } \\
\text { traps }\end{array}$ & $\begin{array}{c}\text { Average density } \\
\text { (the number of adults / } \\
\text { sticky traps) }\end{array}$ & $\begin{array}{c}\text { The number of } \\
\text { adults }\end{array}$ & $\begin{array}{c}\text { Attractant percent } \\
(\%)\end{array}$ \\
\hline white & $0.00 \mathrm{a}$ & 0 & 0 \\
yellow & $0.00 \mathrm{a}$ & 0 & 0 \\
green & $2.04 \pm 0.10 \mathrm{e}$ & 110 & 54.46 \\
blue & $1.02 \pm 0.019 \mathrm{~d}$ & 55 & 27.23 \\
red & $0.00 \mathrm{a}$ & 0 & 0 \\
black & $0.00 \mathrm{a}$ & 0 & 0 \\
turquoise & $0.48 \pm 0.049 \mathrm{c}$ & 25 & 12.38 \\
purple & $0.20 \pm 0.019 \mathrm{~b}$ & 12 & 5.94 \\
\hline
\end{tabular}

Note: The letters in the same column in the table show significant differences $(P<0.05)$, while the same letters show no significant difference $(P \geq 0.05)$, the following table is the same.

Trapping effect of different heights of sticky traps on adults. As shown in table 2, there was a significant difference in the effect of different suspension height sticky traps on adult of Cotarina sp. The number of adults trapped by sticky traps with $1.0 \mathrm{~m}$ away from the ground was the greatest, with the attractive effect $37.62 \%$. Followed by $1.5 \mathrm{~m}$ away from the ground, while $0.5 \mathrm{~m}$ away from the ground received the worst trapping effect. 
Table 2 Trapping effects of different heights of sticky traps on adult of Cotarina sp.

\begin{tabular}{cccc}
\hline $\begin{array}{c}\text { Different heights of } \\
\text { sticky traps }\end{array}$ & $\begin{array}{c}\text { Average density } \\
\text { (the number of adults / sticky } \\
\text { trap) }\end{array}$ & $\begin{array}{c}\text { The number } \\
\text { of adults }\end{array}$ & $\begin{array}{c}\text { Attractant percent } \\
(\%)\end{array}$ \\
\hline $1.5 \mathrm{~m}$ & $0.92 \pm 0.024 \mathrm{a}$ & 66 & 32.67 \\
$1.0 \mathrm{~m}$ & $1.07 \pm 0.11 \mathrm{ab}$ & 76 & 37.62 \\
$0.5 \mathrm{~m}$ & $0.82 \pm 0.037 \mathrm{~b}$ & 60 & 29.70 \\
\hline
\end{tabular}

Population dynamics of adult Cotarina sp. The population dynamics of adult Cotarina sp. in the fields were shown in figure 1. The tendency of adult Cotarina sp. attractived by different colors of sticky traps was consistent. The number of the adults slowly increased since $25 \mathrm{March}$, then reached the maximum on 29 March. The number of the adults reached the second peak on 3 April.

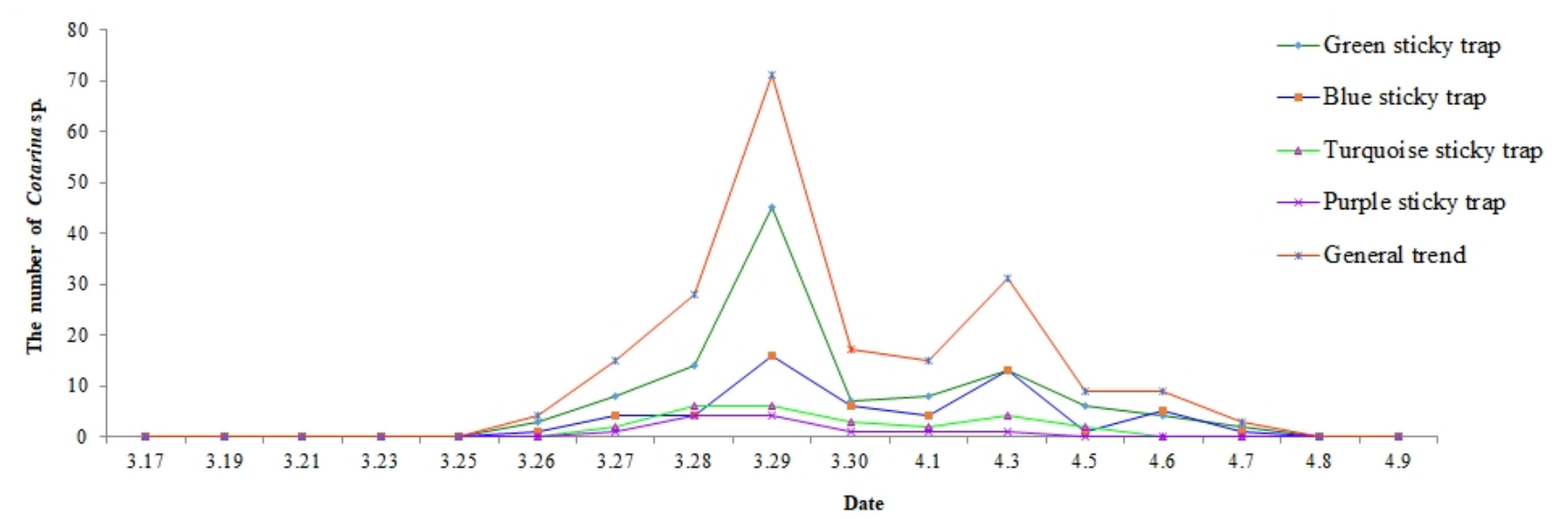

Figure 1 The population dynamics of adult Cotarina sp. in the field

\section{Conclusions and Discussions}

Cotarina sp. is commonly known as "the mulberry fruit gall-midge", and it is principal pests on mulberry since the early $80 \mathrm{~s}^{[3]}$. Since then, it began to rapidly spread around the mulberry planting area. However, at present, the control of Cotarina sp. is still mainly dependent on chemical prevention treatment while other measures used less. This paper is the first time to monitor of the mulberry fruit gall-midge.

The results showed that yellow sticky traps had no trapping effect on adult Cotarina sp. in open field, which was different with the other species of Diptera insect in China ${ }^{[5]}$. Green sticky traps had extremely excellent trapping effect on adult, followed by was blue sticky traps, while turquoise and purple sticky traps received worst trapping effects. The adults had no taxis to yellow, red, black, and white sticky traps. Those may be related to the hanging position of the sticky traps and overwintering population base of Cotarina sp.. Because in the early stage of the experiment, we investigated the development progress of Cotarina sp., found that the population of the area hanging green sticky traps was larger than other area hanging other color sticky traps.

The results showed that the number of adults trapped by sticky traps with $1.0 \mathrm{~m}$ away from the ground was the greatest, followed by $1.5 \mathrm{~m}$ away from the ground, while $0.5 \mathrm{~m}$ away from the ground received the worst trapping effect. The trapping effect is affected by many factors, such as the biological characteristics of Cotarina sp., the growth and development of mulberry and so on. In this test, effects of three different hanging heights on trapping effect were significantly different, and the sticky trap with the middle to the ground received the best trapping effect, possibly because adult Cotarina sp. had poor activity and feeding habits with water. The specific reasons need further study and determination. 
In conclusion, the results of this experiment have shown that the suspension height and the color of the sticky traps had significant impacts. The adults of Cotarina sp. had certain tendency to the green, blue, purple, turquoise sticky traps. The green sticky traps showed most attractive effect than other colors. The number of adults trapped by sticky traps with $1.0 \mathrm{~m}$ away from the ground was the greatest. The tendency of adult Cotarina sp. attractived by different colors of sticky traps were consistent. Two peaks were found in an emergence curve for the adults in spring, first peak on $29 \mathrm{March}$, the second on 3 April.

\section{References}

[1] P. Reichao, Z. Panpan, Z. Aichun: Biological characteristics and chemical control of the mulberry fruit gall midge Cotarina sp., Journal of Plant Protection, 2015, 42(3): 304 - 309.

[2] J. Feng, H. Mingzhai, W. Shuxa: Studies on the biological characters and control of Contarinia morulae Jiang Nomen Nudum (Diptera: Cecidomyiidae), Science of Sericulture, 1997, 23(2): 73 - 76.

[3] H. Mingzhai, J. Feng, W. Shuxia: A preliminary report on the study of morphological characters and life habit in mulberry sorosls gall-midge, Shanxi Sericulture, 1994(2): 12 - 13.

[4] C. Zhe, X. Fangling, X. Lihua: Attractive effect of different color sticky traps and suspension ways on blueberry drosophila, Forest Pest and Disease, 2017, 36(2): 13 - 15.

[5] Z. Huaijiang, W. Pengxiang, L. Xiaoxia: Attractive effect of the different colored sticky traps on Dasyneura pyri (Bouch), South China Fruits, 2015, 44(4): 93 -95. 